2. P. J. Cohen, The independence of the continuum hypothesis, Proc. Nat. Acad. Sci. U. S. A. 50 (1963), 1143-1148; 51 (1964), 105-110.

3. H. J. Keisler, Ultraproducts and saturated models, Indag. Math. 26 (1964), 178186.

4. M. Morley and R. Vaught, Homogeneous universal models, Math. Scand. 11 (1962), 37-57.

5. A. Tarski and R. Vaught, Arithmetical extensions of relational systems, Compositio Math. 13 (1957), 81-102. 313.

6. R. Vaught, Models of complete theories, Bull. Amer. Math. Soc. 69 (1963), 299-

7. - A Löwenheim-Skolem theorem for cardinals far apart, Proceedings of Symposium on Model Theory, Berkeley, Calif., June-July, 1963 (to appear).

University of California, Los Angeles

\title{
A REMARK ON WIENER'S TAUBERIAN THEOREM
}

M. KAC

A recent note by Levinson [1] made it seem worthwhile to point out that a weaker version of the Tauberian theorem can be proved in a few lines which is, however, strong enough to provide a proof of the prime number theorem.

Let $K(x) \in L(-\infty, \infty)$ and assume that its Fourier transform obeys the standard condition

$$
\begin{aligned}
\kappa(\xi) & =\int_{-\infty}^{\infty} K(x) e^{i \xi x} d x \\
& \neq 0 \text { for all }-\infty<\xi<\infty .
\end{aligned}
$$

One version of Wiener's Tauberian theorem is the assertion that if $m(y)$ is a bounded measurable function such that for almost all $x$,

$$
\int_{-\infty}^{\infty} K(x-y) m(y) d y=0
$$

then $m(y)=0$ almost everywhere.

The weaker version of the Tauberian theorem is obtained by adding an extra requirement on the function $K(x)$, namely that

$$
x^{2} K(x) \in L(-\infty, \infty) .
$$

Received by the editors October 10, 1964. 
To use this to prove the prime number theorem, we can follow the proof given by Levinson, since here one had

$$
K(x)=\left\{\begin{array}{cc}
0 & \text { for } x \leqq 0 \\
R\left(e^{x}\right) e^{-x} & \text { for } x>0
\end{array}\right.
$$

where $R$ is a bounded function; condition (3) is thus satisfied with "plenty to spare."

To prove the weaker version, consider the class $\Phi$ of functions $\phi$ which have a continuous second derivative and which vanish outside a bounded interval. Let $\phi(\xi) \in \Phi$, and set

$$
F(x)=\int_{-\infty}^{\infty} \phi(\xi) e^{i x \xi} d \xi
$$

Clearly, $F(x) \in L(-\infty, \infty)$, and $|F(x)||K(x-y)||m(y)|$ is integrable as a function of $(x, y)$, where $K$ and $m$ obey the hypotheses above. Hence, using (2) and Fubini's theorem, we have

$$
\begin{aligned}
0 & =\int_{-\infty}^{\infty} F(x)\left(\int_{-\infty}^{\infty} K(x-y) m(y) d y\right) d x \\
& =\int_{-\infty}^{\infty} m(y)\left(\int_{-\infty}^{\infty} K(x-y) F(x) d x\right) d y
\end{aligned}
$$

and clearly

$$
\int_{-\infty}^{\infty} K(x-y) F(x) d x=\int_{-\infty}^{\infty} \kappa(\xi) \phi(\xi) e^{i \xi y} d \xi .
$$

Thus, for each function $\phi$ in $\Phi$, we will have

$$
0=\int_{-\infty}^{\infty} \int_{-\infty}^{\infty} m(y) \kappa(\xi) \phi(\xi) e^{i \xi \nu} d \xi d y .
$$

The stronger requirement (3) on $K(x)$ implies that its transform $\kappa(\xi)$ has a continuous second derivative; since, by assumption (1), $\kappa(\xi)$ is never zero, we see that multiplication by $\boldsymbol{\kappa}$ carries the class $\Phi$ into itself exactly: $\kappa \Phi=\Phi$. We can rewrite (7) as

$$
0=\int_{-\infty}^{\infty} m(y)\left(\int_{-\infty}^{\infty} \phi(\xi) e^{i \xi y} d \xi\right) d y
$$

for every function $\phi$ in the class $\Phi$. Since $\Phi$ is closed under translation, we can replace $\phi(\xi)$ by $\phi(\xi-\alpha)$ and apply the usual change of variable to arrive at 


$$
0=\int_{-\infty}^{\infty} m(y)\left(\int_{-\infty}^{\infty} \phi(\xi) e^{i \xi y} d \xi\right) e^{i \alpha y} d y
$$

holding now for all real $\alpha$. Using (4), this may be written as

$$
0=\int_{-\infty}^{\infty} m(y) F(y) e^{i \alpha y} d y
$$

for all real $\alpha$. By the uniqueness of Fourier transforms, we may conclude that

$$
m(y) F(y)=0
$$

for almost all $y$.

Since $\phi$ has compact support, $F(y)$ is an entire function, and can be chosen not to be identically zero. Since it can then have at most a denumerable number of zeros, $m(y)=0$ for almost all $y$, and the proof is complete.

It should perhaps be pointed out that the proof above uses implicitly the concept of a generalized Fourier integral (forced upon us by the fact that $m(y)$ is merely bounded). Also, the relation $\boldsymbol{\Phi}=\Phi$ is somewhat reminiscent of the algebraic nature of the Tauberian theorem.

\section{REFERENCE}

1. N. Levinson, The prime number theorem from $\log n$ !, Proc. Amer. Math. Soc. 15 (1964), 480-485.

The Rockefeller INSTItUTE 